\title{
The influence of pressure on the structure and dynamics of hydrogen bonds in zoisite and clinozoisite
}

\author{
Björn Winkler ${ }^{1}$, Julian D. Gale ${ }^{2}$, Keith Refson ${ }^{3}$, \\ Daniel J. Wilson ${ }^{1}$, Victor Milman ${ }^{4}$ \\ ${ }^{1}$ Institut für Geowissenschaften, Universität Frankfurt, Frankfurt a.M., Germany \\ ${ }^{2}$ Nanochemistry Research Institute, Curtin University, Perth, WA \\ ${ }^{3}$ Rutherford Appleton Laboratory, ISIS Facility, Didcot OX11 0QX, Oxon, England \\ ${ }^{4}$ Accelrys, 334 Science Park, Cambridge CB4 0WN, UK
}




\begin{abstract}
Density functional theory calculations have been used to study the pressure-induced changes of the hydrogen bond of Fe-free orthozoisite and clinozoisite and the concomitant shifts of the $\mathrm{OH}$ stretching frequencies. Two independent parameter-free lattice dynamical calculations have been employed. One was based on a plane wave basis set in conjunction with norm-conserving pseudopotentials and a density functional perturbation theory approach, while the other used a localised basis set and a finite displacement algorithm for the lattice dynamical calculations. Both models confirm the unusually large pressure-induced red-shift found experimentally $\left(-33.89 \mathrm{~cm}^{-1} / \mathrm{GPa}\right)$ in orthozoisite, while the pressure-induced shifts in clinozoisite are much smaller $\left(-5--9 \mathrm{~cm}^{-1} / \mathrm{GPa}\right)$. The atomistic model calculations show that in orthozoisite the nearly linear $\mathrm{O}-\mathrm{H} \cdots \mathrm{O}$ arrangement is compressed by about $8 \%$ on a pressure increase to $10 \mathrm{GPa}$, while concomitantly the $\mathrm{O}-\mathrm{H}$ distance is significantly elongated (at $10 \mathrm{GPa}$ by $2.5 \%$ ). In clinozoisite, the $\mathrm{O}-\mathrm{H} \cdots \mathrm{O}$ arrangement is kinked $\left(\angle \mathrm{OHO}=166^{\circ}\right)$ at ambient conditions and remains kinked at high pressures, while the O-H distances is elongated by $0.5 \%$ at $10 \mathrm{GPa}$ only. The current calculations confirm that correlations between the distances and dynamics of hydrogen bonds, which have been established at ambient conditions, cannot be used to infer hydrogen positions at high pressures.
\end{abstract}

Keywords: A. Zoisite; B. High pressure; C. Hydrogen bond; D. lattice dynamics.

\title{
1 Introduction
}

Hydrogen bonds are important structural features in a very large number of minerals, and accordingly have been studied extensively (see e.g. references in Farmer [1974], Rossman [1988], Libowitzky [1999]). The large volume of data available on both static structural aspects, derived from diffraction experiments, and of dynamic aspects, generally obtained from infrared spectroscopy, has allowed to establish consistent correlations between the atomic arrangement of atoms in a hydrogen bond and its spectroscopic signature. A review of earlier work and a new derivation of such correlations has been presented by Libowitzky [1999]. These correlations are thought to provide a key to the understanding of hydrogen bonds in those systems in which the positions of the hydrogen atoms cannot be determined directly, but for which IR spectroscopy, with which the dynamics of ppm levels of hydrogen bonds can be measured, has been employed to obtain the stretching and bending frequencies. This was a major ad- 
vance in the understanding of the incorporation of hydrogen into nominally anhydrous minerals (NAM), which are thought to provide a reservoir of hydrogen in the deep Earth.

In the past, the interpretation of IR spectra was generally phenomenological, i.e. the band assignments were based on comparisons to other structures. This is generally unproblematic for the identification of hydrogen bonds, for which, in silicates, the stretching and bending vibrations are usually well separated from the vibrations of the other structural building blocks. This approach, however, does not allow to unravel details of the atomic arrangement when several hydrogen bonds are present or when the environment is unusual. A step beyond the 'fingerprint' method is the use of lattice dynamical calculations based on an empirical description of the interatomic interactions. Such lattice dynamical calculations based on potential models give qualitatively correct results, but their predictive power is rather limited. Recently, parameter-free model calculations, based on density functional perturbation theory, DFPT, or finite displacements algorithms, have been shown to be very accurate and reliable, and can provide insight into structure-property relations not available otherwise. Therefore, it is now possible to use such approaches to clarify unusual observations and interpret spectra more rigorously. Here we present a density functional based study on zoisite, with the aim of deepening our understanding of the hydrogen bond in this system.

Zoisite, $\mathrm{Ca}_{2} \mathrm{Al}_{2}\left(\mathrm{Al}_{1-p} \mathrm{Fe}_{p}\right)\left[\mathrm{O}|\mathrm{OH}| \mathrm{Si}_{2} \mathrm{O}_{7} \mid \mathrm{SiO}_{4}\right]$, is the orthorhombic (space group Pnma) iron-poor end-member of the epidote group. Its physical properties have been studied extensively, and have recently been reviewed, together with comparisons to other epidote group minerals [Liebscher and Franz, 2004]. One structural feature which has attracted attention in the past is the single symmetrically independent hydrogen-bond of intermediate strength with an $\mathrm{OH}$ stretching frequency of $\nu(\mathrm{OH}) \approx 3150$ $\mathrm{cm}^{-1}$ at ambient pressure. This hydrogen bond has been studied extensively, mainly by vibrational spectroscopy. A review of these studies has recently been presented by Liebscher [2004]. The influence of pressure on the $\mathrm{OH}$ valence vibration in zoisite has been studied up to $11.6 \mathrm{GPa}$ by infrared spectroscopy [Winkler et al., 1989]. In that study, it was found that $\nu(\mathrm{OH})$ shifts linearly to lower wave numbers, where the shift was found to be $-33.9 \mathrm{~cm}^{-1} / \mathrm{GPa}$. Qualitatively, this is the expected behaviour, as on compression the hydrogen bond is shortened, leading to a lengthening and weakening of the O-H bond, with a concomittant decrease of $\nu(\mathrm{OH})$. However, a comparison to pressure-induced frequency shifts of $\mathrm{OH}$ bonds observed in other minerals, as will be discussed in the following, suggests that the shift in zoisite is anomalously large. 
In structurally related clinozoisite a later study Bradbury and Williams [2003] found a pressure induced shift of only $-5.1(9) \mathrm{cm}^{-1} / \mathrm{GPa}$. Bradbury and Williams [2003] discuss possible origins of the difference of the pressure-induced behaviour of the hydrogen bond of clinozoisite and zoisite. They note that in zoisite the hydrogen bond at ambient condition is stronger $\left(\nu(\mathrm{OH})_{\mathrm{zoi}}=3170 \mathrm{~cm}^{-1}, \nu(\mathrm{OH})_{\mathrm{clino}}\right.$ $=3351 \mathrm{~cm}^{-1}$ ) shorter and straighter, and that this may be the origin of the rather different pressure behaviour. While the compressibilities reported for zoisite and clinozoisite scatter significantly (see summary in [Gottschalk, 2004]), a combination of experimental data and results of ab initio calculations now clearly shows that clinozoisite (bulk modulus $\mathrm{B} \approx 142 \mathrm{GPa}$ ) is less compressible than zoisite $(\mathrm{B} \approx$ $129 \mathrm{GPa}$ ). It is, however, not obvious why this difference in the complressibility should lead to such a significant change in the pressure-dependence of the frequency of the $\mathrm{OH}$-stretching vibration.

A comparison with pressure-induced shifts in other minerals also suggests that the findings for orthozoiste are unusual. For brucite, $\mathrm{Mg}(\mathrm{OH})_{2}$, the pressure-induced red-shift of the $\mathrm{OH}$-stretching vibration is $-0.6 \mathrm{~cm}^{-1} / \mathrm{GPa}$ [Kruger et al., 1989], while isostructural portlandite, $\mathrm{Ca}(\mathrm{OH})_{2}$, shows a shift of -2.1 $-3.5 \mathrm{~cm}^{-1} / \mathrm{GPa}$ [Kruger et al., 1989, Shinoda et al., 2000]. In katoite, $\nu(\mathrm{OH})$ remains constant at about $3660 \mathrm{~cm}^{-1}$ up to pressures of about $5 \mathrm{GPa}$. There are also examples where the $\mathrm{OH}$-stretching frequency shifts to higher energies with increasing pressure. For example, in nominally anhydrous coesite, $\mathrm{SiO}_{2}$, hydrogen can be incorporated through the hydrogarnet substitution and pressure-induced blue shifts of $\approx 7 \mathrm{~cm}^{-1} / \mathrm{GPa}$ have been observed [Koch-Müller et al., 2003]. In chloritoids, the two symmetrically distinct hydrogen bonds behave very differently, as the high energy one displays a pressure-induced blue shift of $\approx 5 \mathrm{~cm}^{-1} / \mathrm{GPa}$ while the second $\mathrm{O}-\mathrm{H}$ stretch, corresponding to a hydrogen bond of intermediate strength $\left(\nu(\mathrm{OH})_{\text {chloro }} \approx 2990 \mathrm{~cm}^{-1}\right)$ shifts to lower energies by $-11 \mathrm{~cm}^{-1} / \mathrm{GPa}$ [Koch-Müller et al., 2002]. Nearly all hydrogen stretching frequencies in serpentine minerals shift to higher wavenumbers (by about $1-10 \mathrm{~cm}^{-1} / \mathrm{GPa}$ ) [Auzende et al., 2004]. In topaz the pressure-induced shift is to higher energies, by $0.6(3) \mathrm{cm}^{-1} / \mathrm{GPa}$ [Bradbury and Williams, 2003]. From this summary it is clear, that the observed shift in orthozoisite is anomalously large.

An elucidation of the pressure-induced structural changes of the hydrogen bond system in structures as complex as zoisite by high pressure diffraction experiments was not possible at the time of the first experiments, and even today a high pressure study at $10 \mathrm{GPa}$ would probably not result in the localisation of the hydrogen atom. At ambient pressure, it is generally straightforward to characterize hydrogenbonds by single crystal x-ray diffraction or by neutron diffraction studies. Even at ambient pressure, 
the use of powder x-ray diffraction for the localisation of hydrogen atoms in low symmetry compounds usually requires advanced refinement techniques. In high pressure experiments the resolution is generally much more limited and the signal-to-noise ratio is significantly decreased in comparison to experiments at ambient pressure. For complex structures, diffractograms obtained by high-pressure powder x-ray diffraction are therefore of insufficient quality to obtain the hydrogen positions by Rietveld refinement. Powder neutron diffraction at high pressures is generally performed on high flux, medium resolution diffractometers. This has been sufficient to obtain hydrogen positions in high symmetry compounds such as garnets [Lager et al., 2005] or compounds with small unit cells, such as brucite [Parise et al., 1994]. However, a high pressure powder neutron diffraction study of a low symmetry, large unit cell compound like zoisite would very likely not give reliable hydrogen positions. While at least in principle, high-pressure single crystal neutron diffraction studies would allow to determine the hydrogen positions (e.g. using neutron Laue diffraction [McIntyre, GJ and Mélési, L and Guthrie, M and Tulk, C A and $\mathrm{Xu}, \mathrm{J}$ and Parise, JB, 2005]), such studies are in their infancy. Hence, while there are some studies of the detailled atomic arrangement of hydrogen bonds at high pressure in structures with small unit cells and/or high symmetry, only very little data is available for complex silicates, and hence reliable atomistic model calculations can provide new data.

Quantum mechanical atomistic models have been used extensively in the past to study the compression behaviour of inorganic compounds (see Winkler [2004] and Tse [2004] and references therein). Currently, most parameter-free atomistic model calculations of inorganic crystalline compounds are based on density functional theory [Winkler, 1999]. Density functional theory has been shown to reliably be able to reproduce, and, as it is a parameter-free approach, predict the compression behaviour and hydrogen bonding [Milman and Winkler, 2001]. Clearly, there are limitiations to this approach due to the necessary approximations for the exchange and correlation interaction. Further inaccuracies are introduced due to implementation-dependent aspects, such as the use of pseudopotentials. In addition, in studies addressing the structure and dynamics of hydrogen bonds, additional problems my arise. Hydrogen is a very light particle and hence the anharmonic contribution in the lattice dynamics is often relatively large [Szalay et al., 2002, Pascale et al., 2004, Tosoni et al., 2005]. Also, in dynamical studies, it may be necessary to go beyond a classical description of the hydrogen nucleus, e.g. by path integral methods [Gillan, 1988]. However, for most silicates, DFT calculations in the athermal limit will provide reliable predictions with sufficient accuracy to allow us to deepen our understanding of the investigated 
process on an atomistic level.

Specifically, developments in the last decade now allow the extension of DFT-based calculations to obtain the lattice dynamics at high pressures. Several methods have been developed for the calculation of vibrational frequencies of solids. In the 'finite displacement approach' the dynamical matrix for the supercell is found by imposing small displacements of one atom at a time, and calculating the forces exerted on all atoms. This allows to obtain the dynamical matrix. In a system with symmetry, the symmetry elements may used to deduce related force constants, thus minimizing the number of simulations that need to be performed. One major limitation of this method is that periodic boundary conditions do not permit a dipole in the unit cell, as this would necessitate a non-periodic potential. At the zone centre, longitudinal optic (LO) phonons in polar materials produce such a dipole, and thus are not correctly calculated; they are instead found to be degenerate with transverse optic (TO) modes. One significant advantage of the finite displacement method is that it can be employed in conjunction with all approaches with which energies and forces can be computed. This allows its use with very accurate methods, such as all electron full-potential linearized augmented plane wave methods, or with other methods employing a localized basis set, such as implemented in the SIESTA code, which has been used here. Furthermore, in plane wave / pseudopotential methods it can be used with computationally efficient ultrasoft pseudopotentials. Finally, the treatment of metallic systems is unproblematic.

The DFPT-approach (see Baroni et al. [2001], Refson et al. [2006] and references therein) has the advantage that it can be used to compute vibrational frequencies at any given point in reciprocal space with a computational effort similar to that required for a calculation at the $\Gamma$-point, i.e. in the long wavelength limit. It can also be used to obtain the LO/TO splitting. There are some complexities in the implementation, so that the use of computationally efficient ultrasoft pseudopotentials is currently not yet available in CASTEP. Instead we have used norm-conserving pseudopotentials. This implies very high cut-offs for the kinetic energy (on the order of $700 \mathrm{eV}$ ) to achieve numerical convergence, which makes these calculations costly. In contrast to the finite displacement method, the application of DFPT to metals with partially occupied bands requires special care. We have recently compared the results of lattice dynamical calculations based on these two different approaches with one DFT implementation for diaspore, and, as expected, the results of these two approaches are very similar [Friedrich et al., 2006]. In the following, we confirm that the different approaches also lead to very similar results for implementations using very different basis sets and for rather complex structures. This then allows us 
to confidently interpret the high pressure infrared spectrum of zoisite, and to understand the pressureinduced structural changes of the hydrogen bond.

Another very interesting feature in the lattice dynamics of zoisite, which has been discussed controversially, is the origin of a band at around $2200 \mathrm{~cm}^{-1}$ in the infrared spectrum. The discussion regarding the origin of this band has been summarized by Liebscher [2004]. It has been debated whether this band is a fundamental $\mathrm{OH}$-stretching vibration of a very strong hydrogen bond, or a combination or an overtone. From deuteration experiments it is clear that this band involves a hydrogen displacement.

The present theoretical lattice dynamical study of zoisite therefore is aimed at confirming the experimentally observed large red-shift of the $\mathrm{OH}$-valence vibration with parameter-free atomistic model calculations and to provide insight into the structural changes responsible for this behaviour.

\section{Computational details}

Density functional theory calculations have been used previously to discuss the relative stability of ironfree clinozoisite and orthozoisite [Winkler et al., 2001] and their compressibilities. The present calculations go significantly beyond that work, as here two independent implementations of a density functional theory-based model have been used, and, secondly, the lattice dynamics of the structures have been investigated.

All SIESTA [Soler et al., 2002] calculations have been performed within the framework of non-local density functional theory using the generalized gradient approximation of Perdew, Burke and Ernzerhof [Perdew et al., 1996]. Here the core electrons and nuclei are represented by non-local pseudopotentials of the Kleinmann-Bylander form, and the construction was performed according to the modified scheme of Troullier and Martins [1991] with inclusion of relativistic effects. A small core pseudopotential was generated for calcium with a valence configuration of $3 p 64 s 2$, with partial core corrections also being included for this element.

The valence electron wavefunction is expanded as a linear combination of strictly localized pseudoatomic orbitals (i.e. these are numerical tabulations of the solutions of the pseudized atomic problem within a confinement potential). The confinement radii were chosen such that the energy of each orbital was increased by $0.005 \mathrm{Ry}$. In the present work, the soft confinement scheme of Junquera et al. [2001] has been employed to avoid the discontinuity in the basis function first derivative at the radial cutoff. 
Here the parameters of the soft-confinement potential are $50 \mathrm{Ry}$ for the prefactor and the radius at which the potential begins to be applied was set to 0.8 times the orbital radius. The basis set employed was of triple- $\zeta$ plus double polarization (TZ2P) quality for all elements. The split-norm value that determines the division of the orbital into different " $\zeta$ " was set at 0.25 , except for hydrogen where the higher value of 0.5 was employed based on previous observations that this yields superior results due to the greater degree of variation in the effective spatial extent of hydrogen with charge state. Similarly, it was found that a modified treatment of the polarisation functions for $\mathrm{Al}$ and $\mathrm{Si}$ was advantageous. The radii for the two $3 d$ orbitals were fixed at 5 and 6 Bohr in both cases, while the soft confinement potential was increased to $100 \mathrm{Ry}$ and the inner radius set to 0 Bohr. The evaluation of Hartree and exchange-correlation potentials was conducted on an auxiliary basis set consisting of a uniform real-space grid truncated by an equivalent kinetic energy cutoff for which a value of $250 \mathrm{Ry}$ was found to be sufficiently precise. For the Brillouin zone integration, a Monkhorst-Pack mesh [Monkhorst and Pack, 1976] was utilized based on a consistent real space truncation radius of $12 \AA$ [Moreno and Soler, 1992]. This corresponds to mesh sizes of $3 \times 5 \times 3$ and $2 \times 5 \times 3$ for clinozoisite and orthozoisite, respectively. Minimisations of all structures were performed using conjugate gradients with an atomic force tolerance of $0.01 \mathrm{eV} / \AA$ and a pressure convergence criterion for unit cell optimisation of 200 bar. Once optimised, the phonons for both polymorphs were computed by central finite differencing of the analytic first derivatives with a displacement of 0.04 Bohr. Similarly the Born effective charge tensors for all atoms were determined by using the same finite difference procedure with respect to the polarisation computed using the Berry phase approach [King-Smith and Vanderbilt, 1993].

The second set of DFT calculations used the pseudopotential/plane-wave method [Payne et al., 1992] and density-functional perturbation theory (DFPT) [Baroni et al., 2001, Gonze, 1997, Refson et al., 2006] to calculate the vibrational spectrum. The plane-wave basis set is unbiassed (as it is not atom-centred) and does not suffer from the problem of basis-set superposition error (BSSE) unlike atom-centred basis sets. It also makes converged results straightforward to obtain in practice as the convergence is controlled by a single adjustable parameter. However these advantages can only be fully realised if the error introduced by the pseudopotential approximation is small. In this study we used norm-conserving pseudopotentials generated using the optimised method of Rappe et al. [1990] as implemented in the OPIUM code (http://www.sourceforce.net). This allows pseudopotentials to be tuned for accuracy and quantitatively assessed for transferrability. The set of pseudopotentials used are generated using configurations 
from the Bennett and Rappe library, and when tested in simple oxides yield lattice parameters whithin around 0.25 calculations. As with the SIESTA calculations it was necessary to treat the $\mathrm{Ca} 3 s$ and $3 p$ states as explicit valence electrons with a "small-core" pseudopotential.

All pseudopotentials were generated using the PBE XC functional [Perdew et al., 1996] to allow fully consistent treatment of the core and valence electrons. Fuchs et al. [1998] showed that inconsistent calculations with an LDA treatment of core electrons and GGA valence electrons led to lattice parameters and bond lengths typically closer to the LDA than the GGA result. This explains why the earlier calculations [Winkler et al., 2001] yielded shorter bond lengths than experiment.

Geometry optimization and lattice dynamics calculations were performed using the CASTEP code [Segall et al., 2002, Clark et al., 2005, Refson et al., 2006]. A plane-wave cutoff of $700 \mathrm{eV}$ was used, and Brillouin-Zone integrals were performed using a 1×3×2 Monkhort-Pack grid (Monkhorst and Pack, 1976). The PBE (Perdew et al, 1996) XC functional was used consistently for pseudopotential generation and plane-wave calculations. Concurrent geometry optimization of unit cell and internal co-ordinates was performed so that forces were converged to $0.005 \mathrm{ev} / \mathrm{A}$ and stress residial to $0.005 \mathrm{GPa}$. Experience shows that a high degree of force convergence is necessary for accurate lattice dynamics calculations. Dynamical matrices were calculated using DFPT in the harmonic approximation and diagonalised to yield frequencies, eigenvectors, and Born effective charges. This is sufficient information to also allow the calculation of infrared absorption coefficients.

\section{Results}

The lattice parameters obtained in the present study are compared to experimental results in Tab. 1 and 2 for orthozoisite and clinozoisite, respectively. For orthozoisite, the values obtained from the two model calculations are in very good agreement with each other as they differ by less than $0.5 \%$. The agreement with experiment is in the expected range, i.e. there is a slight overestimation by about $1 \%$ for each lattice parameter. For clinozoisite, we have calculated the structure with SIESTA only, and again we obtain a slight overestimation of the lattice parameters with respect to the epxerimental values of about $1 \%$. The main difference between the current plane wave-based calculations and an earlier study [Winkler et al., 2001] is the use of norm-conserving pseudopotentials in the present study, while ultrasoft pseudopotentials generated with the LDA were used before. This leads to minor changes in the structural parameters. 
For example, experimentally it is found that three $\mathrm{Si}-\mathrm{O}$ distances in the coordination tetrahedra of $\mathrm{Si}(2)$ have approximately the same length $(1.62 \AA)$, while the fourth $\mathrm{Si}-\mathrm{O}$ distance is significantly shorter $(1.58$ $\AA$ ). Here and in the following, the labelling of Dollase [1968] has been employed. In the older study, the distortion was reproduced, but the bonds were systematically $\approx 0.03 \AA$ too short. In the present study, the distortion is again well reproduced, but the $\mathrm{Si}-\mathrm{O}$ distances are now systematically $\approx 0.018 \AA$ too long. The SIESTA calculations also reproduce the distortion of the tetrahedra, here the overestimation is $\approx 0.025 \AA$. Other unusual details of the structure are equally well described. The unusual $97^{\circ} \mathrm{O}-\mathrm{Si}-\mathrm{O}$ bond in the $\mathrm{Si}(3)$-coordination polyhedra [Dollase, 1968] has an angle of $96^{\circ}$ in the relaxed structure obtained from the SIESTA calculation and $97^{\circ}$ from the CASTEP calculation.

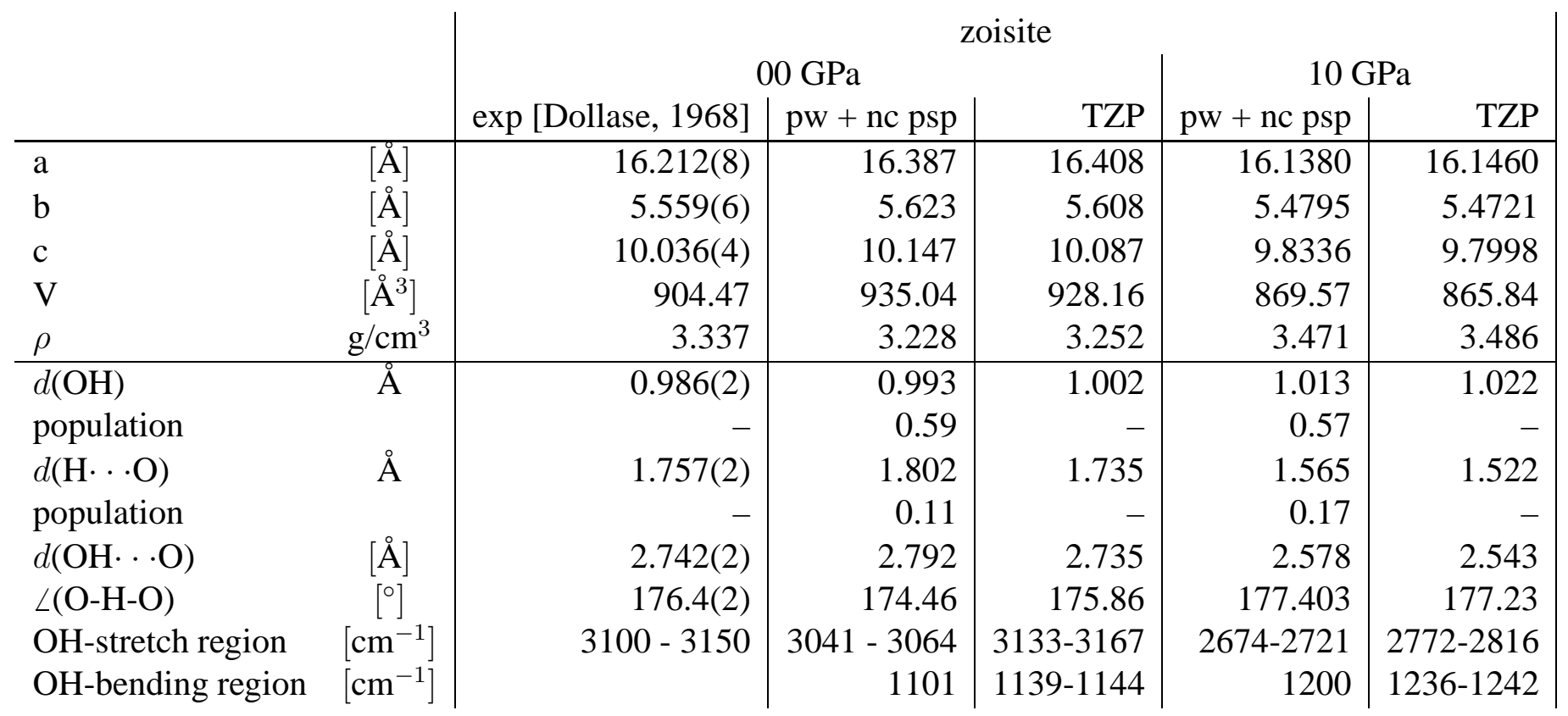

Table 1: Structural parameters for the hydrogen bond are from a low-temperature neutron diffraction study [Smith et al., 1987]. X-ray data from Comodi and Zanazzi [1997] and Smith et al. [1987] give a significantly different $\mathrm{O}-\mathrm{H}-\mathrm{O}$ angle of $\approx 169^{\circ}$.

A more detailed analysis of the hydrogen environment for orthozoisite is given in Table 1 . At 0 GPa the two models give an $\mathrm{OH}$ distance of $\approx 0.997 \AA$, they agree within each other to better than $1 \%$. They are in good agreement with the single crystal neutron diffraction results of Smith et al. [1987], who obtained $d(\mathrm{OH})=0.986(2) \AA$. The $\mathrm{OH}$ distance obtained by $\mathrm{x}$-ray diffraction by Dollase $[1968](d(\mathrm{OH})$ $=1.25 \AA$ ) is improbable. The calculated $\mathrm{O}-\mathrm{O}$ distance in both models agrees with the experimental values to within $1 \%$, which differ by about $0.008 \AA$ between different refinements [Smith et al., 1987, Comodi and Zanazzi, 1997, Dollase, 1968]. There are some small differences between the results of the two models regarding the hydrogen bond length, but they are both in good agreement with experiment. A 


\begin{tabular}{lc|r|r|r|} 
& & \multicolumn{3}{|c|}{ clinozoisite } \\
& & exp [Kvik et al., 1988] & \multicolumn{2}{|c}{ TZP } \\
& & $00 \mathrm{GPa}$ & $00 \mathrm{GPa}$ & $10 \mathrm{GPa}$ \\
\hline $\mathrm{a}$ & {$[\AA]$} & $8.893(2)$ & 8.9709 & 8.7854 \\
$\mathrm{~b}$ & {$[\AA]$} & $5.630(3)$ & 5.6397 & 5.5095 \\
$\mathrm{c}$ & {$[\AA]$} & $10.150(2)$ & 10.1958 & 9.9312 \\
$\beta$ & {$[\AA]$} & $115.36(1)$ & 115.26 & 115.03 \\
$\mathrm{~V}$ & {$\left[\AA^{3}\right]$} & 459.21 & 466.51 & 435.56 \\
$\rho$ & $\mathrm{g} / \mathrm{cm}^{3}$ & 3.280 & 3.235 & 3.464 \\
\hline$d(\mathrm{OH})$ & {$[\AA]$} & 0.9753 & 0.9940 & 0.9988 \\
$d(\mathrm{H} \cdots \mathrm{O})$ & {$[\AA]$} & $1.964(1)$ & 1.873 & 1.699 \\
$d(\mathrm{OH} \cdots \mathrm{O})$ & {$[\AA]$} & $2.922(1)$ & 2.850 & 2.679 \\
$L(\mathrm{O}-\mathrm{H}-\mathrm{O})$ & {$\left[{ }^{\circ}\right]$} & $166.9(1)$ & 166.77 & 165.89 \\
\hline$\nu(\mathrm{OH})$ & {$\left[\mathrm{cm}^{-1}\right]$} & 3327 & 3319,3328 & 3224,3242
\end{tabular}

Table 2: Comparison of experimental data from neutron diffraction experiments at $15 \mathrm{~K}$ [Kvik et al., 1988] and results from calculations using the SIESTA program.

comparison of the computed $\mathrm{O}-\mathrm{H}-\mathrm{O}$ angle to experiment is interesting, as there is a significant difference between the results of the neutron diffraction study [Smith et al., 1987], where the O-H-O angle was determined to be $176.4^{\circ}$, while the $\mathrm{x}$-ray studies consistently give a much lower value of $\approx 161-169^{\circ}$ [Dollase, 1968, Comodi and Zanazzi, 1997, Smith et al., 1987]. Clearly, the present calculations support the findings derived from the neutron diffraction experiment. In summary, this analysis confirms the results of Milman and Winkler [2001], who have shown that quantum mechanical models such as those used here give a realistic description of the hydrogen bond systems, which is often superior to that obtained from than x-ray studies.

This point of view is strengthened by a comparison of the structural data for clinozoisite, where the O-H distances derived from single crystal x-ray data sets [Dollase, 1968, Comodi and Zanazzi, 1997] are unrealistic. In the X-ray structure refinement of Comodi and Zanazzi [1997], $d(\mathrm{OH})=0.683 \AA$, $d(\mathrm{OH} \cdots \mathrm{O})=2.892 \AA$ and an $\mathrm{O}-\mathrm{H}-\mathrm{O}$ angle $=163.4^{\circ}$, Dollase [1968] reports $d(\mathrm{OH})=0.769 \AA$ and $d(\mathrm{OH} \cdots \mathrm{O})=2.89 \AA$ A. Neutron diffraction data [Kvik et al., 1988] again provide a more reliable benchmark. The calculations agree well with the experimentally determined $\mathrm{OH}$-distance and the O-H-O angle. The hydrogen bond length, however, is underestimated in the model by about $4.5 \%$ and the $\mathrm{OH} \cdots \mathrm{O}$ distance is too short by $2.5 \%$. However, it is worthwhile to note that in the structure derived from the neutron diffraction data the hydrogen position is not as well defined as the other structural parameters, similar to the neutron diffraction results for orthozoisite. The atomic displacement parameters for hydrogen are two- to three-times larger than those of the other atoms. Also, the sample studied contained an 
appreciable amount of iron, which very likely will influence the hydrogen position. In absolute terms, the differences between experiment and theory are small and this again strengthens the argument that approaches such as those used here can be used to investigate hydrogen bonds in complex structures.

The comparison of the calculated $\mathrm{OH}$-stretching frequencies at ambient conditions to experimental values (Table 1 and 2 ) shows that the wave numbers are reproduced to within $\approx 80 \mathrm{~cm}^{-1}$. For orthozoisite, the average frequency of $\nu(\mathrm{OH})$ differs by approximately $100 \mathrm{~cm}^{-1}$ between the two models at ambient pressure, as it is $\approx 3050 \mathrm{~cm}^{-1}$ for the plane wave calculation and $3150{ }^{-1}$ for the SIESTAbased model. The experimental value for $\nu(\mathrm{OH})$ is $3100-3150 \mathrm{~cm}^{-1}$. As has been mentioned above, the SIESTA calculations have not been corrected for LO/TO-splitting. Also, no corrections for anharmonicity have been applied in either calculation. From an experimentalists point of view, $80 \mathrm{~cm}^{-1}$ is a very significant difference. However, in relative terms, this is a difference of only $2.5 \%$, which is about the same accuracy one observes for low frequency phonons [Refson et al., 2006, Dubinin et al., 2004]. Similarly, for clinozoisite the computed $\mathrm{OH}$-stretching frequencies are in very good agreement with the experimentally determined one (Tab. 2). Clearly, the near perfect agreement is fortuitous, as no corrections have been applied to the values obtained from the model. DFT-based calculations are known to reliably describe the compression behaviour of silicates, and as the accuracy of DFPT calculations at high pressures will be the same as at ambient pressure, the findings described until now allow us to predict the high pressure behaviour of the hydrogen bond, for which no structural data is available.

For orthozoisite, both models predict that the $\mathrm{OH}$ bond will be stretched by $2 \%$ if the pressure is increased to $10 \mathrm{GPa}$. Concomitantly, the hydrogen bond decreases by $15 \%$ in the CASTEP calculations and by $12 \%$ in the SIESTA calculations. The values for the $\mathrm{H} \cdots \mathrm{O}$ distances at $10 \mathrm{GPa}$ agree to within $3 \%$ between the two models. At $10 \mathrm{GPa}$, the results differ also by $100 \mathrm{~cm}^{-1}$ between the two models, i.e. the difference is about as large as it has been for the ambient pressure calculation. The pressure induced shift is in both models the same, namely $-35 \mathrm{~cm}^{-1} / \mathrm{GPa}$. This is in excellent agreement with the one experimental value reported for orthozoisite, namely $-33.9 \mathrm{~cm}^{-1}$ [Winkler et al., 1989]. For clinozoisite, the model predicts a shift of $-9 \mathrm{~cm}^{-1} / \mathrm{GPa}$. This is higher than the experimentally determined value of $-5.1(9) \mathrm{cm}^{-1} / \mathrm{GPa}$ [Bradbury and Williams, 2003]. However, at $10 \mathrm{GPa}$ the difference in the computed and experimentally determined pressure-dependence would result in an overestimation of the stretching frequency of only $40 \mathrm{~cm}^{-1}$, which is a small value, given the numerous sources of error. For example, the magnitude of the shift is pressure dependent, and in the experimental study [Bradbury and Williams, 
2003] the range over which the data were fitted was $16 \mathrm{GPa}$. A reanalysis of the experimental data to $10 \mathrm{GPa}$ yields a slightly larger shift of about $6 \mathrm{~cm}^{-1} / \mathrm{GPa}$. The current calculations refer to a iron-free end-member composition, while the experiment was performed with an iron-containing sample, and as the iron contents seems to influence the hydrogen position, this likely will contribute to the differences between model calculations and experiments. Also, at high pressures, the bands become very broad, and that leads to difficulties in defining the background and peak positions. However, one can confidently conclude that despite these small discrepancies between the models and the models and experiment the calculations clearly confirm that the hydrogen bond in orthozoisite is anomalous in that the pressureinduced shift of the stretching frequency is at least three times larger than in clinozoisite and about $6-7$ times large than the shift generally observed for pressure-induced shifts.

Band assignments in the past have generally been based on phenomenological approaches and crystal chemical reasoning. It has generally been assumed that bands can be associated to be dominated by motions of distinct entities, such as 'asymmetric $\mathrm{SiO}_{4}$ stretching' and the like. The current calculations yield the dynamical matrices and hence the complete eigenvectors are available. A symmetry analysis is hence straightforward and the irreducible representation of a specific mode can easily be determined. However, in the case of low symmetry compounds with hundreds of modes this is not really helpful. The results of lattice dynamical calculations are then best analysed by a straightforward visualisation of the modes by viewers such as the commercial 'Material Studio' software or the public domain software GDIS [Fleming and Rohl, 2005]. Such visualisations are instructive, as they generally show that the simplified view of characterising modes according to their presumed 'molecular' or 'coordination polyhedra' character are not really describing the dynamics well. In the case of clinozoisite, for example, the $\mathrm{OH}$-stretching vibrations can be easily identified. The next 8 phonons in the frequency range of 1107 $1028 \mathrm{~cm}^{-1}$ can be reasonably well described as asymmetric $\mathrm{SiO}_{4}$-stretches and (at 1074 and $1064 \mathrm{~cm}^{-1}$ ) OH-bending. Below this frequency, it is not obvious that any 'molecular' vibration dominates the distortion patterns. For example, at $934 \mathrm{~cm}^{-1}$ there is a mode which clearly involves the hydrogen atoms, but also all $\mathrm{SiO}_{4}$-tetrahedra are distorted significantly.

On increasing pressure the $\mathrm{OH}$-bending vibrations in clinozoisite shift from 1074 and $1064 \mathrm{~cm}^{-1}$ to 1132 and $1123 \mathrm{~cm}^{-1}$. This shift of nearly $6 \mathrm{~cm}^{-1} / \mathrm{GPa}$ is of opposite sign as the shift of the $\mathrm{OH}$-stretching vibration. For orthozoisite, the $\mathrm{OH}-$ bending vibrations are calculated to have energies of 1139 - 1144 $\mathrm{cm}^{-1}$ (SIESTA) or $1101 \mathrm{~cm}^{-1}$ (CASTEP). On pressure increase, both models calculations predict a blue 
shift of $9.8 \mathrm{~cm}^{-1} / \mathrm{GPa}$. So, also for the $\mathrm{OH}$-bend there is an appreciably larger shift in orthozoisite than in clinozoisite.

In clinozoisite, the ' $\mathrm{SiO}_{4}$-stretching frequencies' at wave numbers $>1000 \mathrm{~cm}^{-1}$ show a pressure induced blue shift of about $5 \mathrm{~cm}^{-1} / \mathrm{GPa}$. This is in good agreement with the experimental findings of Bradbury and Williams [2003], who obtain values of 3.6(8) and 4.5(8) $\mathrm{cm}^{-1} / \mathrm{GPa}$. In orthozoisite, the CASTEP calculations give a blue shift of about $4 \mathrm{~cm}^{-1} / \mathrm{GPa}$. Hence, these modes seem to behave similarly in ortho- and clinozoisite with respect to their pressure-dependence.

\section{Discussion}

We have used two rather different implementations of density functional theory based models, where one is based on a 'conventional' plane wave / pseudopotential approach, while the second uses a technique which has, due to the use of a local basis set, other advantages and limitations. In addition, the lattice dynamical calculations were based on different approaches, namely a DFPT approach for the calculations using a plane wave basis set and a finite displacement algorithm in the SIESTA calculations. This has led, as was expected, to some discrepancies in the absolute values for the structural and physical properties, but it is gratifying to see that the predicted magnitudes of changes are very close.

Based on the two independent parameter-free atomistic models, the present study has unambiguously confirmed that the hydrogen bond in orthozoisite is 'anomalous' in the sense that the pressure-induced red-shift is about six or seven times as large as the red-shifts observed in other silicates. This conclusion is strengthened by the results for clinozoisite, which give a much smaller (by a factor of at least three) shift, although the latter is nearly twice as large as the experimental value.

The current study allows to correlate the pressure-induced shift quantitatively to changes in the geometry of the hydrogen bonds. It is now clear that the nearly linear hydrogen bond in orthozoisite remains linear on compression, while the kinked arrangement in clinozoisite remains kinked on pressure increase. Both experimentally [Kvik et al., 1988] and from our model calculations it is found that at ambient conditions the hydrogen bond in clinozoisite is bend by more than $30^{\circ}$ (angle $\left.(\mathrm{OH} \cdots \mathrm{O}) \approx 167^{\circ}\right)$ and it seems from the model calculations that this deviation from linearity tends to increase with increasing pressure, although here further calculations are required to confirm this trend. Hence, the calculations presented here imply that the interpretation of the origin of the large half width of the $\mathrm{OH}$ stretching 
vibration in clinozoisite is likely not as straightforward as proposed by Bradbury and Williams [2003], who take the hydrogen bond in clinozoisite to be 'almost linear' and 'straightforwardly compacting'. In fact their method of analysis seems to be much more applicable to orthozoisite instead.

There has been a long-standing discussion on whether or not there is a second hydrogen bond in orthozoisite. This was first suggested by Langer and Lattard [1980], based on an unusual band at 2170 $\mathrm{cm}^{-1}$, wich was assigned to the $\mathrm{OH}$-stretching vibration of a short hydrogen bond. In a neutron diffraction study there was 'no diffraction evidence of a second proton position' [Smith et al., 1987], although the authors cautioned that that may be due to the resolution of their experiment. More recently Liebscher et al. [2002] interpreted the band at $2170 \mathrm{~cm}^{-1}$ as an overtone of the bending vibration of a second, bifurcated hydrogen bond between $\mathrm{O}(10)-\mathrm{H} \cdots \mathrm{O}(2)$. In the high pressure study of Winkler et al. [1989] it was shown, that the pressure-induced shift of the $2170 \mathrm{~cm}^{-1}$ band was rather large $\left(\approx 19 \mathrm{~cm}^{-1} / \mathrm{GPa}\right)$. Liebscher et al. [2002] dispute the assignment of Winkler et al. [1989], who proposed that this band is an overtone of the bending vibration of the $\mathrm{O}(10)-\mathrm{H} \cdots \mathrm{O}(4)$ hydrogen bridge. Their argument is that one would expect the overtone to be at $1950-2000 \mathrm{~cm}^{-1}$ and that Winkler et al. [1989] did not discuss why this band is absent in the structurally related clinozoisite. Liebscher et al. [2002] state that polarized single crystal spectra would help resolving the controversy. These have been measured by Winkler [1988] and seem to indicate, that the $2170 \mathrm{~cm}^{-1}$ band is due to the same dipole as the 'conventional' stretching motion, i.e. by the $\mathrm{O}(10)-\mathrm{H}$ group.

As the current calculations do not allow a straightforward computation of two-phonon processes, an unambiguous clarification of the origin of the $2170 \mathrm{~cm}^{-1}$ is not possible. However, the current calculations clearly show that the bending motion of the hydrogen bridge between the $\mathrm{O}(10)-\mathrm{H} \cdots \mathrm{O}(4)$, for which there is unambiguous evidence from diffraction, has an energy of about $1140 \mathrm{~cm}^{-1}$. Hence, neglecting any corrections for anharmonicity, the first overtone would be at $2280 \mathrm{~cm}^{-1}$. Anharmonic corrections would likely lead to a decrease of the frequency of the overtone with respect to a doubling of the fundamental frequency, and hence the model calculations are consistent with the assignment of Winkler et al. [1989]. A second argument for the assignment by Winkler et al. [1989] is that here we compute a shift of about $9 \mathrm{~cm}^{-1}$, and hence the second overtone would, again neglecting any second order effects, shift by twice that value. This is in near perfect agreement with the observed value of $\left(\approx 19 \mathrm{~cm}^{-1} / \mathrm{GPa}\right.$ ) [Winkler et al., 1989]. As the observed shift is so large, it is unlikely that this band results from a combination of an $\mathrm{OH}-$ bending motion with a high-frequency phonon involving the $\mathrm{SiO}_{4^{-}}$ 
tetrahedra, as the pressure-induced shift of the latter is generally found to be too small to lead to such a large resultant shift. So, from the available evidence, it seems that the most straightforward explanation which rests only on well-established structural data is an assignment of the $2170 \mathrm{~cm}^{-1}$ band to the overtone of the $\mathrm{OH}$-bending vibration. There is no straightforward answer to the question why this vibration does not occur in clinozoisite. However, absorption cross-sections are very sensitive to structural details and hence this may be the cause of the absence of the $2170 \mathrm{~cm}^{-1}$ band in clinozoisite. One approach to clarify the assignement would be by quantum mechanical molecular dynamics. However, such calculations are computationally demanding, and while the algorithms are well established, a simulation with a reasonably sized supercell for an appreciable time is currently still a grand challenge project. A second method, which we are currently pursuing, is to compute the NMR spectrum and compare it to experiment.

More generally, the current study demonstrates the accuracy with which the dynamics of hydrogen bonds can be investigated, even if corrections for anharmonicity are neglected. It is well established that these are important in studies relying on an optimal agreement with experimental values, but for the study of relative values, such as pressure-induced shifts, a straightforward approach seems to suffice.

The present study also confirms the earlier finding [Friedrich et al., 2006] that it is not advisable to use the correlations between structural parameters and lattice dynamics established at ambient pressure to infer atomic positions from IR data at high pressures. These correlations are very well established at ambient pressure (see e.g. Libowitzky [1999] and references therein). However, fig. 1 shows that there is a linear change of the $\mathrm{OH}$-stretching frequency as a function of the pressure-induced shortening of the distance $d(\mathrm{H} \cdots \mathrm{O})$, and not, as the correlation would predict, and exponential decrease. The results shown here and those obtained by Friedrich et al. [2006] imply that any derivation of structural parameters from infrared data based on the correlations derived at ambient pressure to would lead to systematically too short $\mathrm{O} \cdots \mathrm{H}$ and $\mathrm{O}-\mathrm{H} \cdots \mathrm{O}$ distances. However, further calculations on hydrogen bonds of different strengths are required to finalize this conclusion. Such calculations are currently in progress.

\section{Acknowledgements}

This research was supported by Deutsche Forschungsgemeinschaft (Project Wi-1232), which is a part of the HydroMin collaborative research project with the EuroMinScI EUROCORES, funded by the ESF 


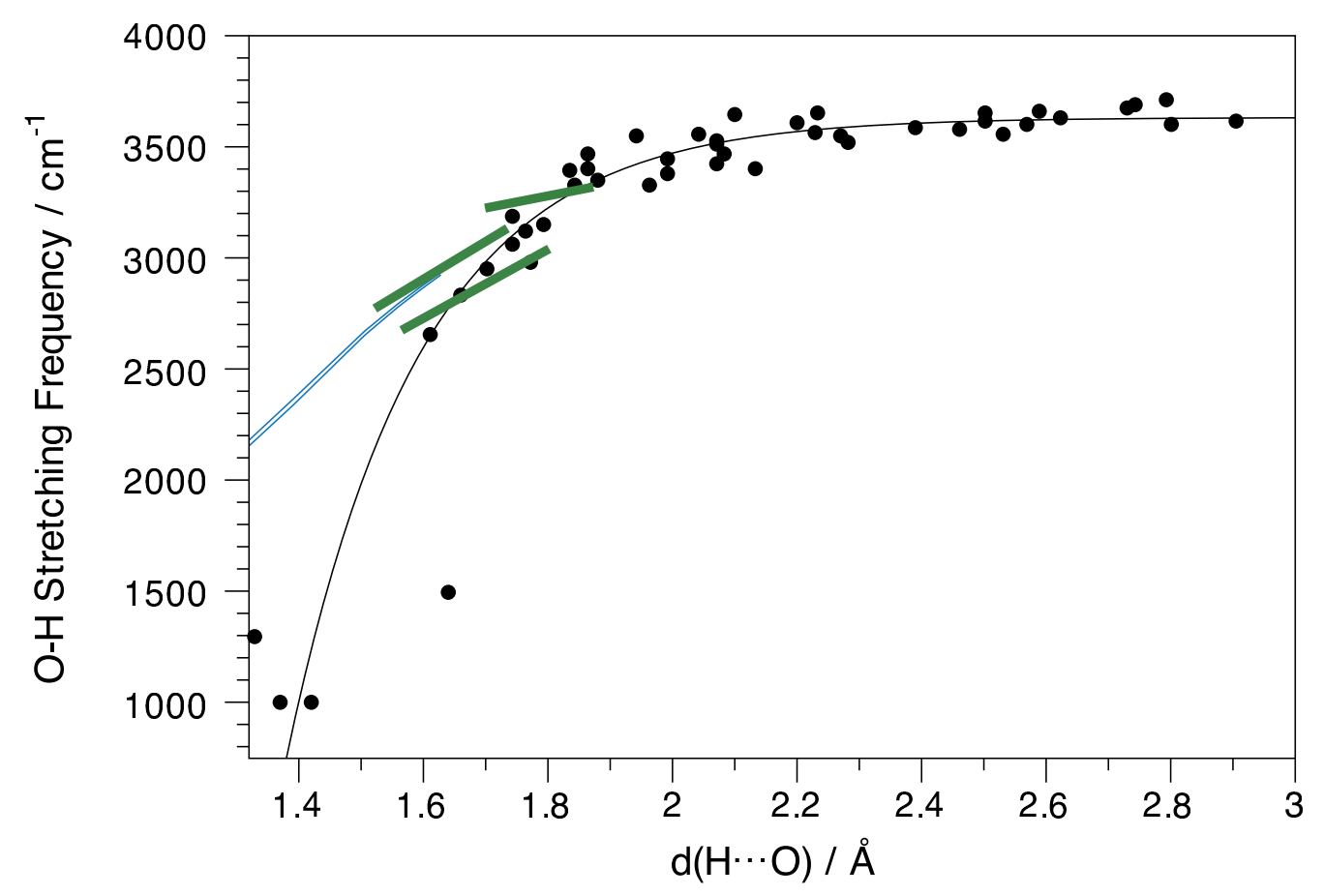

Figure 1: Correlation between the distance $d(\mathrm{H} \cdots \mathrm{O})$ and the $\nu(\mathrm{OH})$-stretching frequency. The points and the full line are taken from [Libowitzky, 1999]. The thin blue line represents the pressure induced shift of $\nu(\mathrm{OH})$ from diaspore [Friedrich et al., 2006]. The three thick lines represent the shift of $\nu(\mathrm{OH})$ in clino- and orthozoisite, where the latter is obtained from two independent calculations (see text).

with funds from the EU sixth framework programme under contract no. ERAS-CT-2003-980409. JDG would like to thank the Government of Western Australia for funding under the Premier's Research Fellowship program. DJW was funded through the DTI (UK) MaterialGrid consortium.

\section{References}

A-L Auzende, I Daniel, B Reynard, C Lemaire, and F Guyot. High-pressure behaviour of serpentine minerals: a Raman spectroscopic study. Physics and Chemistry of Minerals, 31:269-277, 2004.

S Baroni, S de Gironcoli, A Dal Corso, and P Giannozzi. Phonons and related crystal properties from density-functional perturbation theory. Reviews of Modern Physics, 73:515-562, 2001.

S E Bradbury and Q Williams. Contrasting bonding behaviour of two hydroxyl-bearing metamorphic minerals under pressure: clinozoisite and topaz. American Mineralogist, 88:1460-1470, 2003. 
S J Clark, M D Segall, C J Pickard, P J Hasnip, M J Probert, K Refson, and M C Payne. First principles methods using CASTEP. Zeitschrift für Kristallographie, 220:567-570, 2005.

P Comodi and P F Zanazzi. The pressure behaviour of clinozoisite and zoisite: An x-ray diffraction study. American Mineralogist, 82:61-68, 1997.

W A Dollase. Refinement and comparison of the structures of zoisite and clinozoiste. American Mineralogist, 53:1882-1898, 1968.

A Dubinin, B Winkler, K Knorr, and V Milman. Lattice dynamics and elastic properties of $\mathrm{PbF}_{2}$ and $\mathrm{BaF}_{2}$ from quantum mechanical calculations. European Physical Journal, B 39:27-33, 2004.

V C Farmer, editor. The infrared spectra of minerals. Mineralogical Society, London, 1974.

S Fleming and A Rohl. GDIS: a visualization program for molecular and periodic systems. Zeitschrift für Kristallographie, 220:580-584, 2005.

A Friedrich, D J Wilson, E Haussühl, B Winkler, W Morgenroth, K Refson, and V Milman. Highpressure properties of diaspore, $\mathrm{AlO}(\mathrm{OH})$. Physics and Chemistry of Minerals, 2006. DOI 101007/s00269-006-0135-5.

M Fuchs, M Bockstedte, E Pehlke, and M Scheffler. Pseudopotential study of binding properties of solids within generalized gradient approximations: The role of core-valence exchange correlation. Physical Review, B 57:2134-2145, 1998.

MJ Gillan. The quantum simulation of hydrogen in metals. Philosophical Maganzine A, 58:257-283, 1988.

X Gonze. First-principles responses of solids to atomic displacements and homogeneous electric fields: Implementation of a conjugate gradient algorithm. Physical Review, B55:10337-10354, 1997.

M Gottschalk. Thermodynamic properties of zoisite, clinozoisite and epidote. In A Liebscher and G Franz, editors, Epidotes, number 56 in Reviews in Mineralogy \& Geochemistry, pages 83-124. Mineralogical Society of America, 2004.

J Junquera, O Paz, D Sanchez-Portal, and E Artacho. Numerical atomic orbitals for linear-scaling calculations. Physical Review, B 64, 2001. 
RD King-Smith and D Vanderbilt. Theory of polarization of crystalline solids. Physical Review, B 47: $1651-1654,1993$.

M Koch-Müller, A M Hofmeister, Y Fei, and Z Liu. High-pressure IR-spectra and the thermodynamic properties of chlorotoid. American Mineralogist, 87:609-622, 2002.

M Koch-Müller, P Dera, Y Fei, B Reno, N Sobolev, E Hauri, and R Wysoczanski. $\mathrm{OH}^{-}$in natural coesite. American Mineralogist, 88:1436-1445, 2003.

M B Kruger, Q Williamns, and R Jeanloz. Vibrational spectra of $\mathrm{Mg}(\mathrm{OH})_{2}$ and $\mathrm{Ca}(\mathrm{OH})_{2}$ under pressure. Journal of Chemical Physics, 91:5910-5915, 1989.

$\AA ̊$ Kvik, J J Pluth, J W Richardson Jr, and J V Smith. The ferric ion distribution and hydrogen bonding in epidote: a neutron diffraction study t 15 K. Acta Crystllographica, B 44:351-355, 1988.

G A Lager, W G Marshall, X Liu, and R T Downs. Re-examination of the hydrogarnet structure at high pressure using neutron powder diffraction and infrared spectroscopy. American Mineralogist, 90:639-644, 2005.

$\mathrm{K}$ Langer and D Lattard. Identification of a low energy oh-valence vibration in zoisite. American Mineralogist, 65:779-783, 1980.

E Libowitzky. Correlation of the O-H stretching frequencies and O-H...O hydrogen bond lengths in minerals. Monatshefte für Chemie, 130:1047-1059, 1999.

A Liebscher. Spectroscopy of epidote minerals. In A Liebscher and G Franz, editors, Epidotes, volume 56 of Reviews in Mineralogy, chapter 3, pages 125-170. Mineralogical Society of America, Washington, D.C., 2004.

A Liebscher and G Franz, editors. Epidotes, volume 56 of Reviews in Mineralogy. Mineralogical Society of America, Washington, D.C., 2004.

A Liebscher, M Gottschalk, and G Franz. The substitution $\mathrm{Fe}^{3+}-\mathrm{Al}$ and the isosymmetric displacive phase transition in synthetic zoisite: A powder x-ray and infrared spectroscopic study. American Mineralogist, 87:909-921, 2002. 
McIntyre, GJ and Mélési, L and Guthrie, $\mathrm{M}$ and Tulk, $\mathrm{C}$ A and $\mathrm{Xu}$, J and Parise, JB. One picture says it all - high-pressure cells for neutron Laue diffraction on VIVALDI. Journal of Physics: Condensed Matter, 17:S3017-S3024, 2005.

V Milman and B Winkler. Prediction of hydrogen positions in complex structures. Zeitschrift für Kristallographie, 216:99-104, 2001.

H J Monkhorst and J D Pack. Special points for Brillouin-zone integration. Physical Review B, 13: 5188-5192, 1976.

J Moreno and JM Soler. Optimal meshes for integrals in real-space and reciprocal space unit cells. Physical review, B45:13891-13898, 1992.

J B Parise, K Leinenweber, Weidner D J, and Tan K. Pressure-induce H bonding: neutron diffraction study of brucite, $\operatorname{Mg}(\mathrm{OD})_{2}$ to 9.3 GPa. American Mineralogist, 79:193-196, 1994.

F Pascale, S Tosoni, C Zicovich-Wilson, P Ugliengo, R Orlando, and R Dovesi. Vibrational spectrum of brucite, $\mathrm{Mg}(\mathrm{OH})_{2}$ : a periodic ab initio quantum mechanical calculation including $\mathrm{OH}$ anharmonicity. Chemical Physics Letters, 396:308-315, 2004.

M C Payne, M P Teter, D C Allan, T A Arias, and J D Johannopoulos. Iterative minimisation techniques for ab initio total energy calculations - molecular dynamics and conjugate gradients. Review of Modern Physics, 64:1045-1097, 1992.

J P Perdew, K Burke, and M Ernzerhof. Generalized gradient approximation made simple. Physical Review Letters, 77:3865-3868, 1996.

A M Rappe, K M Rabe, E Kaxiras, and Joannopoulos J D. Optimized pseudopotentials. Physical review, B 41:1227-1230, 1990.

K Refson, PR Tulip, and SJ Clark. Variational density-functional perturbation theory for dielectrics and lattice dynamics. Physical Review B, 73, 2006.

G R Rossman. Vibrational spectroscopy of hydrous components. In Hawthorne F C, editor, Spectroscopic Methods in Mineralogy and Geology, volume 18 of Reviews in Mineralogy, pages 193-206. Mineralogical Society of America, Washington, D. C., 1988. 
M D Segall, P J D Lindan, M J Probert, C J Pickard, P J Hasnip, S J Clark, and M C Payne. Firstprinciples simulation: ideas, illustrations and the CASTEP code. Journal of Physics: Condensed Matter, 14:2717-2744, 2002.

K Shinoda, T Nagai, and N Aikawa. Pressure-dependent anharmonic coefficient of $\mathrm{OH}$ in portlandite by NIR-IR spectroscopy with DAC. Journal of Mineralogical and Petrological Sciences, 95:65-70, 2000.

J V Smith, J J Pluth, J W Richardson Jr, and ÅKvik. Neutron difraction study of zoisite at 15 k and x-ray study at room temperature. Zeitschrift für Kristallographie, 179:305-321, 1987.

JM Soler, E Artacho, JD Gale, A Garcia, J Junquera, P Ordejon, and D Sanchez-Portal. The SIESTA method for ab initio order-N materials simulation. Journal of Physics: Condensed Matter, 14:27452779, 2002.

V Szalay, L Kovács, M Wölecke, and E Libowitzky. Stretching potential and equilibrium length of the OH bond in solids. Chemical Physics Letters, 354:56-61, 2002.

S Tosoni, Pascale F, P Ugliengo, R Orlando, VR Saunders, and R Dovesi. Quantum mechanical calculation of the oh vibrational frequency in crystalline solids. Molecular Physics, 103:2549-2558, 2005.

N Troullier and JL Martins. Efficient pseudopotentials for plane-wave calculations. Physical Review, B43:1993-2006, 1991.

J S Tse. Computational high pressure science. In A Katrusiak and P McMillan, editors, High-pressure Crystallography, volume 140 of NATO Science Series, pages 179-198. Kluwer Academic publisher, 2004.

B Winkler. Introduction ot high pressure computational crystallography. In A Katrusiak and P McMillan, editors, High-pressure Crystallography, volume 140 of NATO Science Series, pages 159-178. Kluwer Academic publisher, 2004.

B Winkler. An introduction to 'Computational Crstallography'. Zeitschrift für Kristallographie, 214: 506-527, 1999. 
B Winkler. OH-Schwingungen in Zoisit: Hochdruck- und polarisierte Einkristallspektren im IR. $\mathrm{PhD}$ thesis, TU Berlin, 1988.

B Winkler, K Langer, and P G Johannsen. The influence of pressure on the $\mathrm{OH}$ valence vibration of zoisite. Physics and Chemistry of Minerals, 16:668-671, 1989.

B Winkler, V Milman, and R H Nobes. A theoretial investigation of the relative stabilities of Fe-free clionzoisite and orthozoisite. Physics and Chemistry of Minerals, 28(471-474), 2001. 